\title{
Stability, mobility and power currents in a two-dimensional model for waveguide arrays with nonlinear coupling
}

\author{
Michael Öster, Magnus Johansson * \\ Department of Physics, Chemistry and Biology (IFM), Linköping University, \\ SE-581 83 Linköping, Sweden
}

\begin{abstract}
A two-dimensional nonlinear Schrödinger lattice with nonlinear coupling, modelling a square array of weakly coupled linear optical waveguides embedded in a nonlinear Kerr material, is studied. We find that despite a vanishing energy difference (Peierls-Nabarro barrier) of fundamental stationary modes the mobility of localized excitations is very poor. This is attributed to a large separation in parameter space of the bifurcation points of the involved stationary modes. At these points the stability of the fundamental modes is changed and an asymmetric intermediate solution appears that connects the points. The control of the power flow across the array when excited with plane waves is also addressed and shown to exhibit great flexibility that may lead to applications for power-coupling devices. In certain parameter regimes, the direction of a stable propagating plane-wave current is shown to be continuously tunable by amplitude variation (with fixed phase gradient). More exotic effects of the nonlinear coupling terms like compact discrete breathers and vortices, and stationary complex modes with non-trivial phase relations are also briefly discussed. Regimes of dynamical linear stability are found for all these types of solutions.
\end{abstract}

Key words: Nonlinear coupling, Peierls-Nabarro potential, mobility, inversion of stability, modulational instability, power currents.

PACS: 42.65.Wi, 42.82.Et, 63.20.Pw, 05.60.-k

* Corresponding author.

Email addresses: micos@ifm.liu.se (Michael Öster), mjn@ifm.liu.se

(Magnus Johansson).

URL: http://people.ifm.liu.se/majoh (Magnus Johansson). 


\section{Introduction}

The use, in optics, of materials with nonlinear responses to external fields has over the past years provided a wealth of applications and prospects for all-optical communication, where light manipulates light itself [1]. Especially, nonlinear photonic structures endowed with a spatially periodic refractive index provide new means to control the flow of light in the form of localized wave packets, not possible in bulk media [2]. The experimental observation of discrete localization in one and two dimensions, in arrays of coupled waveguides experiencing a cubic (Kerr) nonlinearity $[3,4,5,6]$ or a saturable nonlinearity through the photovoltaic effect in a photorefractive medium [7] as well as in optically induced nonlinear photonic lattices $[8,9,10,11]$, shows significant progress in this direction. The theoretical foundation and predictions of these phenomena can, to good approximation, be made by models of the discrete nonlinear Schrödinger (DNLS) type $[6,12,13,14,15,16]$ derived within coupled-mode theory [17] or the tight-binding approximation using Wannier function expansion [18]. The latter technique is also applicable for models of Bose-Einstein condensates trapped in deep optical lattices. In these contexts the issue of mobility of localized packets of energy is highly interesting as it provides insights as how to control optical signals, e.g., for applications in multiport switching.

As is well known [19], travelling localized solutions, or solitons, quite generally exist in continuum systems as a result of the competition between nonlinearity and dispersion. When the continuous symmetry is broken, as is the case when considering a nonlinear discrete system, the generic solution is instead a non-moving time-periodic one, called a discrete breather (DB) or intrinsic localized mode (see, e.g., [20] for a review). The presence of a lattice leads to pinning of localized solutions, as this underlying structure effectively introduces a periodic potential for travelling excitations due to the difference in energy between stationary solutions localized on a lattice site and solutions localized between lattice sites $[21,22]$. This energy difference is referred to as a Peierls-Nabarro (PN) energy barrier and is the reason that narrow travelling excitations get trapped in a lattice. Mobility of a highly localized stationary mode can be induced if an appropriate perturbation is added to overcome the potential barrier, but usually some energy is lost to radiation and the result is trapping $[23,24]$. However, excitations that are broad, in comparison with the lattice spacing, can still propagate as the effects of the PN-barrier in this case is small and the dynamics is well approximated by the continuum limit of the discrete equation. This is provided when the continuum equation has stable localized travelling solutions, which, e.g., is the case for the $1 \mathrm{D}$ cubic nonlinear Schrödinger equation but not for the 2D (cubic) equation [25].

Notable exceptions from the pinning behaviour are integrable lattice equa- 
tions, i.e., equations possessing an infinite number of conserved quantities, like the Ablowitz-Ladik (AL) model which is a discretization of the nonlinear Schrödinger equation retaining this property [26]. These models have analytic exponentially localized travelling solutions and completely lack PN-barrier, which accounts for the excellent mobility. Unfortunately, in contrast to their many interesting mathematical properties, they seem to have very limited direct applications for real systems. Thus other routes to mobility in discrete systems are sought, although these models are conveniently used as the starting point of perturbative approaches. In [27] it is numerically shown how a mobile excitation develops a resonant tail when the governing equation deviates from the 1D integrable AL-model. Moreover, the amplitude of the tail grows when the equation is further away from the integrable limit, or alternatively, as the PN-barrier increases from zero. It is inferred that the effect of pinning is overcome by a periodic exchange of energy between the localized core and the oscillating tail as the excitation moves through the lattice and the periodic PN-potential. This result can also be extended to a $2 \mathrm{D}$ setting [28]. However, there is a limit as to how far the mobile solutions can be continued away from the integrable limit, with broad solutions being more persistent as can be expected [27]. Hence, we can expect that mobility of localized modes is possible when the PN-barrier is small, and the ability to tailor the size of the PN-barrier may allow for a control of the mobility of narrow solutions in a lattice.

Recent research has focused on ways to minimize the PN-barrier, which in optics applications means moving beyond models based on the cubic DNLS equation where the PN-barrier is an increasing function of the power of the stationary modes [4]. Similar to the problem of moving kinks in Klein-Gordon lattices [29] the introduction of competing nonlinearities has proven fruitful. In DNLS equations with saturable nonlinearity the vanishing of the PN-barrier at certain points and a subsequent enhanced mobility has been demonstrated in both 1D [30] and 2D [31], and the same apply for a 1D DNLS model with nonlinear interactions [32]. As these models are derived from real physical settings they are interesting from an application point of view. Note also that there is a large class of DNLS models [33] with an absence of PN-barrier (not only zero at isolated points), different from the more physical models, as the former are special discretizations of the nonlinear Schrödinger model that in some sense preserve a translational invariant [34,35]. Amongst them is the AL-lattice and other integrable equations.

A problem related to the vanishing of the PN-barrier at specific points is that of stability inversion between stationary solutions centred on a lattice point and between lattice points, respectively, which can be understood from the energy minimum principle. The solution with lowest energy at given power will be stable, and the change of sign of the energy difference is also related to an exchange of stability of the lowest energy modes. This can be observed for 
the above models $[30,31,32,36,37]$, as well as for the DNLS model with competing cubic-quintic nonlinearity [38] and more general lattice models [39,40]. In connection with the stability inversion there also exists an intermediate asymmetric solution interpolating between the stationary solutions centred on and in-between sites and bifurcating with these at the points where they change their stability properties $[31,32,38,39,40]$. For the 1 D saturable DNLS model this solution can even be found analytically near the first zero of the PN-barrier [41]. From this viewpoint, the mobility of a highly localized mode may be thought of as a transformation between stationary on- and off-site solutions, via the intermediate solution. Hence a more accurate definition of the PN-barrier would need to include also the intermediate solution as this may be the mode with the highest energy. Therefore, we will distinguish between the energy difference between two fundamental modes and the PN-barrier relating to the energy of all involved stationary modes. Also, in 2D, one would in principle need to consider modes corresponding to different directions of possible propagation [31].

Though localized modes and their mobility are interesting for beam steering in waveguide arrays, transport of energy in the transversal direction (from waveguide to waveguide) can be achieved also by a collective excitation of the waveguides. With a plane wave propagating in the array, the same power will be transported along all waveguides, but there will also be a transfer of power between the waveguides depending on the wave number (phase gradient) of the plane wave. In [42] it is demonstrated that the direction and magnitude of the transversal flow of power in a 1D array with nonlinear coupling are quite versatile under the variation of the amplitude and wave number of the plane waves. Here the discussion is extended to 2D and will show an even greater flexibility.

The outline of the Paper is as follows: In Sec. 2 we present a model for optical waveguides coupled in two spatial dimensions and embedded in a nonlinear Kerr material, discuss its relevance and introduce some of the important properties of the model. We should stress that our model does not attempt to describe configurations where the waveguides themselves are nonlinear, such as those presently experimentally realized in, e.g., $[3,4,5,6,7,8,9,10,11]$, but rather correspond to the original proposal of Jensen [12] with the nonlinearity residing solely in the embedding material. The stability of the fundamental localized modes are investigated in Sec. 3 and their relation to mobility of localized excitations is discussed in Sec. 4. In Sec. 5 some special solutions, like compact and complex modes, arising from a balance of the coupling terms in the model are presented. Finally, in Sec. 6 the transport properties of plane waves are analyzed before we conclude in Sec. 7. We also include in the end of Sec. 7 a brief discussion about some recent related works, which appeared in press after the original submission of this manuscript. 


\section{Model}

The model we consider is a direct extension to two spatial dimensions of a one-dimensional model for the propagation of the electric field amplitudes in coupled optical waveguides embedded in a nonlinear material [32]. The waveguides are identical and regularly spaced in the two spatial dimensions, thus forming a square lattice. Coupling between the sites of the lattice is mediated by an evanescent field overlap of the modes (single-mode propagation) in the respective waveguides and it is assumed that the modes decay sufficiently fast outside the waveguides to motivate only nearest-neighbour coupling. Further, the waveguides themselves are assumed to be constructed from a linear material and surrounded by a nonlinear Kerr material. This will tend to strengthen the effects of nonlinear coupling with respect to on-site nonlinear effects when compared to a system where also the waveguides are nonlinear. This motivates an extension of the generally employed cubic on-site DNLS model to an equation that in two dimensions will read

$$
\begin{aligned}
\mathrm{i} \frac{d \Psi_{n, m}}{d z}= & Q_{1} \Psi_{n, m}+Q_{2} \Delta \Psi_{n, m}+2 Q_{3} \Psi_{n, m}\left|\Psi_{n, m}\right|^{2} \\
& +2 Q_{4}\left[2 \Psi_{n, m} \Delta\left(\left|\Psi_{n, m}\right|^{2}\right)+\Psi_{n, m}^{*} \Delta\left(\Psi_{n, m}^{2}\right)\right] \\
& +2 Q_{5}\left[2\left|\Psi_{n, m}\right|^{2} \Delta \Psi_{n, m}+\Psi_{n, m}^{2} \Delta \Psi_{n, m}^{*}\right. \\
& \left.\quad+\Delta\left(\Psi_{n, m}\left|\Psi_{n, m}\right|^{2}\right)\right],
\end{aligned}
$$

where $\Psi_{n, m}$ is the complex amplitude of the electric field, $z$ measures the propagation along the waveguide array and the coupling parameters $Q_{1}-Q_{5}$ are given by overlap integrals of the modes. The operator $\Delta$ is defined by $\Delta \Psi_{n, m}=\Psi_{n-1, m}+\Psi_{n+1, m}+\Psi_{n, m-1}+\Psi_{n, m+1}$. (Note that the operator $\Delta-4$ is the standard 2D discrete Laplacian.)

The model (1) is also relevant in other contexts, e.g., as a nonlinear tightbinding approximation, with $Q_{4}$ argued to be negligible, for the time-evolution of Bose-Einstein condensates (BEC) in a deep periodic optical potential $[43,44,45]$, or for $Q_{3} / 2=Q_{4}=Q_{5}$ as a rotating-wave approximation to a Fermi-PastaUlam chain [21]. In both these one-dimensional models the extension to two spatial dimensions is straightforward. One may also in a straightforward way consider extensions to other lattice geometries, such as e.g. triangular/hexagonal patterns (which in some sense could be considered as the most natural packing of waveguides in two dimensions). In this work, we chose to consider only the square lattice which is the simplest $2 \mathrm{D}$ structure to analyze from a modelling point of view, and also for comparison with previously published work in the area which mainly dealt with square-lattice configurations. We expect that most of the qualitative results obtained here will persist also for different lattice geometries, although a more detailed discussion on these issues is beyond the scope of the present work. 


\subsection{Parameter estimates}

For general waveguide arrays $Q_{2}>0$ and $Q_{j}, j=3,4,5$, have the same sign as the Kerr index of the nonlinear material. Estimates of the relative strengths of the parameters show that the nonlinear coupling terms, $Q_{4}$ and $Q_{5}$, can be up to the same order of magnitude as the on-site nonlinearity $Q_{3}$ for an array of waveguides constructed from AlGaAs with sizes in the tenth of micrometer regime and operated with a laser in the infrared $(\lambda \approx 1.5 \mu \mathrm{m})$. Details of the derivation of the $1 \mathrm{D}$ model can be found in $[32,46]$ and a calculation of the coupling parameters for a slab waveguide array can be found in Appendix A of [46] (see in particular Fig. A2 in [46] for an explicit illustration of realistic values for relative coupling strengths). When extending the model to two dimensions there is also the possibility of coupling in the diagonal direction between next-nearest neighbours. However, for experimentally relevant sizes, as given above, of an array of square waveguides the linear diagonal coupling can be estimated to be an order of magnitude smaller than the linear direct coupling (see Appendix A2 in [46]). Also, when operated in the power regime where the most interesting phenomena occur, the nonlinear coupling and on-site terms will be of the same order as the linear coupling, i.e. $\left|Q_{2}\right| \sim\left|Q_{j}\right| \sup \left\{\left|\Psi_{n, m}\right|^{2}\right\}, j=3,4,5$. This can be shown to correspond to powers of a few $\mathrm{kW}$ [46], well within what is experimentally available. However, although these estimates of the parameter values are of relevance when considering experimental verifications and applications of the model they will not be taken as a restriction in the present paper, since we are also interested in the general effects of nonlinear coupling.

Further, the parameter $Q_{1}$ can be made to vanish by the transformation $\Psi_{n, m}(z) \mapsto \Psi_{n, m}(z) \mathrm{e}^{-\mathrm{i} Q_{1} z}$ and has no significant physical role. Note also that the staggering transformation $\Psi_{n, m}(z) \mapsto(-1)^{n+m} \Psi_{n, m}(z)$ is equivalent to changing the sign of the parameters $Q_{2}$ and $Q_{5}$, thus reducing the part of parameter space that needs to be investigated to get a complete picture of the equation. Further, by rescaling the amplitudes and the time-like variable, two additional parameters can be fixed. Here we will fix $Q_{2}=0.2$ and $Q_{3}=0.5$, while restricting $Q_{4} \geq 0$ without loss of generality.

\subsection{Conserved quantities}

From Noether's theorem we know that any infinitesimal transformation leaving the action integral for an equation invariant leads to a quantity that is conserved under the evolution governed by that equation [47]. Essentially this means that any continuous symmetry of Eq. (1) corresponds to a conserved quantity. In particular, the invariance under translations in $z$ will lead to con- 
servation of the Hamiltonian $\mathcal{H}=\sum_{n, m} \mathcal{H}_{n, m}$, with

$$
\begin{aligned}
\mathcal{H}_{n, m}=\{ & Q_{2}\left(\Psi_{n, m} \Psi_{n+1, m}^{*}+\Psi_{n, m} \Psi_{n, m+1}^{*}\right)+\frac{Q_{3}}{2}\left|\Psi_{n, m}\right|^{4} \\
+Q_{4}[ & 2\left|\Psi_{n, m}\right|^{2}\left(\left|\Psi_{n+1, m}\right|^{2}+\left|\Psi_{n, m+1}\right|^{2}\right) \\
& \left.+\Psi_{n, m}^{2}\left(\Psi_{n+1, m}^{* 2}+\Psi_{n, m+1}^{* 2}\right)\right] \\
+2 Q_{5}[ & \Psi_{n, m} \Psi_{n+1, m}\left(\Psi_{n, m}^{* 2}+\Psi_{n+1, m}^{* 2}\right) \\
& \left.\left.+\Psi_{n, m} \Psi_{n, m+1}\left(\Psi_{n, m}^{* 2}+\Psi_{n, m+1}^{* 2}\right)\right]\right\}+ \text { c.c. }
\end{aligned}
$$

The evolution equation (1), and its complex conjugate, can be obtained from the Hamilton equations of motion using the canonical variables $\Psi_{n, m}$ and $\mathrm{i} \Psi_{n, m}^{*}$,

$$
\begin{aligned}
\mathrm{i} \frac{d \Psi_{n, m}}{d z} & =\frac{\partial \mathcal{H}}{\partial \Psi_{n, m}^{*}}, \\
-\mathrm{i} \frac{d \Psi_{n, m}^{*}}{d z} & =\frac{\partial \mathcal{H}}{\partial \Psi_{n, m}} .
\end{aligned}
$$

Further, the invariance of Eq. (1) under global phase rotations, i.e., $\Psi_{n, m} \mapsto$ $\Psi_{n, m} \mathrm{e}^{\mathrm{i} \alpha}, \alpha \in \mathbb{R}$, corresponds to the conservation of the norm

$$
\mathcal{N}=\sum_{n, m} \mathcal{N}_{n, m}=\sum_{n, m}\left|\Psi_{n, m}\right|^{2}
$$

Although the Hamiltonian $\mathcal{H}$ for all practical purposes constitutes an energy functional it has no direct connection to any physical energy for the array of waveguides. But from a mathematical viewpoint it can be treated as the energy of the system. In particular, for the cubic DNLS model it holds that a bounded extremum (a maximum in the present formulation since $Q_{2}, Q_{3}>0$ and hence $\mathcal{H}$ should be regarded as negative energy) of $\mathcal{H}$ for given norm $\mathcal{N}$ is Lyapunov stable and may, with right, be called the ground state of the system [48]. This ground state is conjectured to be unique up to a global phase factor, which is consistent with the absence of stability inversion and the non-vanishing PN-barrier in the cubic on-site DNLS model. The norm $\mathcal{N}$, however, has a direct physical interpretation as the total power carried along the waveguides, provided that the modes are appropriately normalized. In the context of BEC, the conservation of norm corresponds to boson number conservation.

The conservation of Hamiltonian and norm may also conveniently be expressed in terms of discrete continuity equations. For this purpose it is instructive to make a change to action-angle variables $\Psi_{n, m}=\sqrt{\mathcal{N}_{n, m}} \mathrm{e}^{-\mathrm{i} \theta_{n, m}}$, where $\mathcal{N}_{n, m}$ and $\theta_{n, m}$ are real canonical variables. The corresponding Hamilton equations 
of motions are

$$
\begin{aligned}
\frac{d \theta_{n, m}}{d z} & =\frac{\partial \mathcal{H}}{\partial \mathcal{N}_{n, m}}, \\
-\frac{d \mathcal{N}_{n, m}}{d z} & =\frac{\partial \mathcal{H}}{\partial \theta_{n, m}} .
\end{aligned}
$$

Now, with $\mathcal{H}_{n, m}$ from Eq. (2) and by introducing the phase difference between neighbouring sites in the two lattice directions as $\varphi_{n, m}=\theta_{n, m}-\theta_{n-1, m}$ and $\phi_{n, m}=\theta_{n, m}-\theta_{n, m-1}$ Eq. (5b) for the norm density can be written as

$$
\frac{d \mathcal{N}_{n, m}}{d z}+\mathcal{J}_{n, m}^{(n)}-\mathcal{J}_{n-1, m}^{(n)}+\mathcal{J}_{n, m}^{(m)}-\mathcal{J}_{n, m-1}^{(m)}=0
$$

The norm current density in the $\hat{\mathbf{n}}$ direction is given by

$$
\begin{aligned}
\mathcal{J}_{n, m}^{(n)}=- & \frac{\partial \mathcal{H}_{n, m}}{\partial \varphi_{n+1, m}}=2 \sqrt{\mathcal{N}_{n, m} \mathcal{N}_{n+1, m}} \sin \varphi_{n+1, m} \\
\times & {\left[Q_{2}+4 Q_{4} \sqrt{\mathcal{N}_{n, m} \mathcal{N}_{n+1, m}} \cos \varphi_{n+1, m}\right.} \\
& \left.+2 Q_{5}\left(\mathcal{N}_{n, m}+\mathcal{N}_{n+1, m}\right)\right]
\end{aligned}
$$

A similar expression holds for $\mathcal{J}_{n, m}^{(m)}=-\partial \mathcal{H}_{n, m} / \partial \phi_{n, m+1}$ in the $\hat{\mathbf{m}}$ direction where the roles of $n$ and $m$ are interchanged. Since the norm density $\mathcal{N}_{n, m}$ is the power on site $(n, m)$, the continuity equation expresses the obvious fact that a change of the power is related to a power transfer to neighbouring sites. Hence the physical interpretation of $\mathcal{J}_{n, m}^{(n)}$ is the flow of power from site $(n, m)$ to site $(n+1, m)$. An equation on the form (6) can also be derived for the Hamiltonian density $\mathcal{H}_{n, m}$, with a Hamiltonian flux density $\mathcal{I}_{n, m}^{(n)}$ and $\mathcal{I}_{n, m}^{(m)}$. For the present purposes it is sufficient to know that for a stationary monochromatic solution, i.e., $\mathcal{N}_{n, m}(z)=\mathcal{N}_{n, m}$ and $\theta_{n, m}(z)=\theta_{n, m}+\Lambda z$, the relation to the norm current density is $\mathcal{I}_{n, m}^{(n)}=\Lambda \mathcal{J}_{n, m}^{(n)}$. Our discussions on the flow of energy in the system (Sec. 6) will thus be made using only the norm current density.

\section{Stationary solutions}

Initially we will turn our attention towards localized solutions or, more specifically, to time-periodic localized modes also known as discrete breathers (DBs) or intrinsic localized modes. The existence of DBs has been rigorously proven for Hamiltonian lattice systems of arbitrary dimension subject to conditions of anharmonicity and non-resonance with linear phonons [49], and extended to more general systems in [50]. In the context of coupled waveguides the variable $z$ will play the role of time. Due to the global phase invariance of the 
DNLS-type equations the time-periodicity leads to a significant simplification of the problem of finding localized solutions. For monochromatic solutions a simple gauge transformation to a rotating frame of reference will render these solutions stationary, i.e., taking $\Psi_{n, m}(z)=\psi_{n, m} \mathrm{e}^{-\mathrm{i}\left(\Lambda+Q_{1}\right) z}$ reduces Eq. (1) to

$$
\begin{aligned}
& -\Lambda \psi_{n, m}+Q_{2} \Delta \psi_{n, m}+2 Q_{3} \psi_{n, m}\left|\psi_{n, m}\right|^{2} \\
& +2 Q_{4}\left[2 \psi_{n, m} \Delta\left(\left|\psi_{n, m}\right|^{2}\right)+\psi_{n, m}^{*} \Delta\left(\psi_{n, m}^{2}\right)\right] \\
& +2 Q_{5}\left[2\left|\psi_{n, m}\right|^{2} \Delta \psi_{n, m}+\psi_{n, m}^{2} \Delta \psi_{n, m}^{*}\right. \\
& \left.+\Delta\left(\psi_{n, m}\left|\psi_{n, m}\right|^{2}\right)\right]=0 .
\end{aligned}
$$

The idea of the existence proofs, and also the foundation for efficient numerical techniques to calculate DBs $[51,52]$, is continuation from the anti-continuous limit, $Q_{j}=0, j=2,4,5$. Solutions are trivial in this uncoupled limit as the amplitude of the oscillators at each site can be chosen independently from the set $\psi_{n, m} \in\left\{0, \pm \sqrt{\Lambda / 2 Q_{3}}\right\}$, where we have made a restriction to real singlefrequency solutions. From the configuration chosen in the anti-continuous limit it is possible to make a classification of the most fundamental localized solutions of Eq. (8). The set of solutions obtained from continuation to non-zero coupling of the configuration with one site excited and all other at rest will be called 1-site DBs. Similarly, if two neighbouring sites are excited at the anti-continuous limit we will get symmetric or anti-symmetric 2-site DBs, depending on the relative phases of the sites.

Since we ultimately are interested in the mobility of localized modes, we would like to compare the Hamiltonian of different stationary solutions. As has been described in Sec. 1 (especially for the corresponding 1D model [32]), the enhanced mobility of localized modes is connected to the vanishing of a PNbarrier measuring the difference in Hamiltonian between fundamental stationary modes. The idea is that for a small or vanishing barrier, mobility of a highly localized mode may be thought of as a continuous transformation between, e.g., stationary 1-site and 2-site solutions, put into motion by adding energy to overcome the barrier (or pinning). Therefore, numerical continuation to non-zero coupling will be performed under the constraint of constant norm. As the parameters $Q_{2}$ and $Q_{3}$ are fixed from rescalings, the parameters $Q_{4}$ and $Q_{5}$ are varied, leaving the frequency $\Lambda$ as a free parameter to be determined by the stationary solution. The choice to keep $\mathcal{N}$ fixed is motivated by the fact that it is a dynamical parameter that is conserved under the evolution of Eq. (1). Hence, when considering a range of coupling parameters (as will shortly be done), this will facilitate a meaningful comparison of the Hamiltonians over that entire range. This would not be the case had the continuation been performed for constant frequency. However, for a fixed frequency and varying norm the relevant energy quantity would instead be the grand canonical free-energy $\mathcal{G}=\mathcal{H}-\Lambda \mathcal{N}$, with $\Lambda$ acting as a chemical potential, as used in $[53]$. 

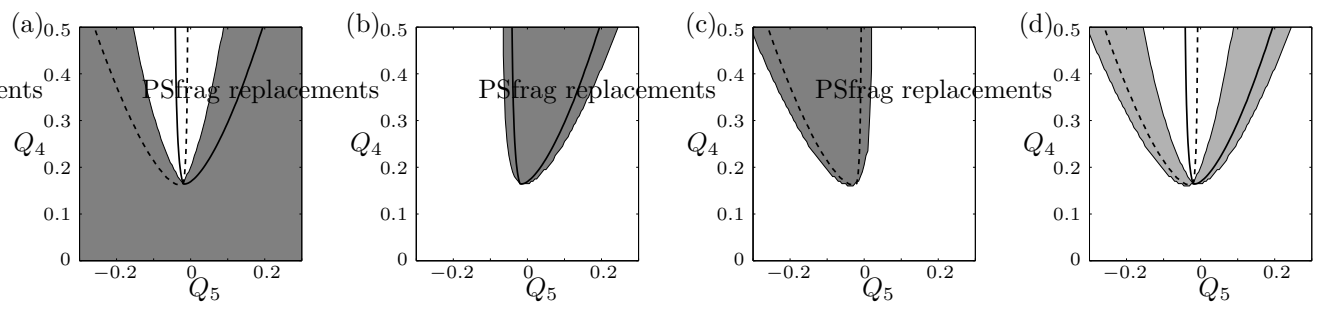

Fig. 1. Region of stability (shaded) for (a) 1-site, (b) symmetric 2-site and (c) anti-symmetric 2-site solution for $Q_{2}=0.2, Q_{3}=0.5$ and $\mathcal{N}=5$; (d) region of existence (shaded) of intermediate solutions (unstable) between the stability boundaries of the 1-site solution and the respective 2-site solution [symmetric (anti-symmetric) in the right (left) region]. Additional small regions of stability, not shown here, can be found for the 2-site solutions for relatively small $Q_{4}$. In the cases of multiple solutions, as for the 1-site case, stability is indicated if at least one solution is stable. See also Figs. 3 and 4 for some details. The solid line in (a), (b) and (d) indicates where the energy difference, $\Delta \mathcal{H}=\mathcal{H}_{1}-\mathcal{H}_{2 s}$, vanishes for the 1-site and symmetric 2-site solutions. The dashed line in (a), (c) and (d) shows the same thing for the 1-site and anti-symmetric 2-site solutions, $\Delta \mathcal{H}=\mathcal{H}_{1}-\mathcal{H}_{2 a}$. The general phenomenology is similar also for other values of the norm $\mathcal{N}$. The size of the lattice is $21 \times 21$ with periodic boundary conditions.

\subsection{Stability}

The linear stability of stationary modes is most simply investigated by studying the evolution of a small perturbation in the rotating frame of reference. Hence, for a given solution we make the ansatz $\Psi_{n, m}(z)=\left[\psi_{n, m}+\left(\epsilon_{n, m}+\right.\right.$ $\left.\left.\mathrm{i} \eta_{n, m}\right) \mathrm{e}^{\lambda z}\right] \mathrm{e}^{-\mathrm{i}\left(\Lambda+Q_{1}\right) z}$ in Eq. (1) and arrive at a linear matrix eigenvalue problem for the growth rates $\lambda$ of the real infinitesimal perturbations $\epsilon_{n, m}$ and $\eta_{n, m}[54]$. Since the problem is Hamiltonian this matrix is infinitesimally symplectic and will have the simultaneous eigenvalues $\pm \lambda, \pm \lambda^{*}$ [55]. Due to the global phase invariance, $\lambda=0$ is always a double eigenvalue. A stationary solution is linearly stable if all eigenvalues are located on the imaginary axis, i.e., we can actually at most make a statement about marginal stability of the solution (meaning that a perturbation at most will grow with a rate that is polynomial in $z$ ).

Extensive calculations, covering a large portion of parameter space, have been done for the most fundamental stationary solutions. In Fig. 1 the regions of stability (shaded) for the 1-site and the two 2-site solutions are shown for fixed norm $\mathcal{N}=5$. For the 1 -site case there actually exist three different solutions in part of the region $Q_{4}>0.165$ (the edge of the instability region), each accessible by continuation but via different paths from the anti-continuous limit. Stability is indicated if one of the solutions is stable (there is no multistability of these three 1-site solutions and some details can also be found in Figs. 3 and 4). The same behaviour appears also in 1D [56], and is presumably an 

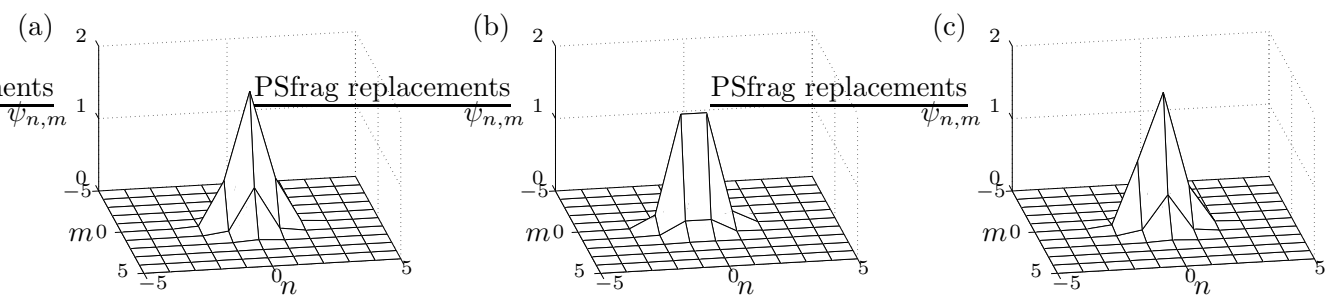

Fig. 2. Examples of solutions for $Q_{2}=0.2, Q_{3}=0.5, Q_{4}=0.25, Q_{5}=0.05$ and $\mathcal{N}=5$. (a) 1 -site solution with $\mathcal{H}=19.7435$ and $\Lambda=7.5019$; (b) symmetric 2-site solution with $\mathcal{H}=19.9278$ and $\Lambda=7.6357$; (c) intermediate solution with $\mathcal{H}=19.7310$ and $\Lambda=7.5036$.

effect of the nonlinear coupling terms that can lead to a vanishing effective coupling between lattice sites. See further the discussion in Sec. 5. From Fig. 1 we see that both stable and unstable solutions of all kinds exist and we note that the stability regions for the 2-site solutions together cover the region of instability of the 1-site solutions. For a quite large range of parameter values we thus have stable propagation of both types of solutions along the array of waveguides. The stability boundaries of the different solutions do not coincide. In the $1 \mathrm{D}$ model $[32,56]$, the stability boundaries very nearly coincide and in fact create a very narrow region of parameter space where the 1-site solution and one of the 2-site solutions have either simultaneous stability or instability. Moreover, the exchange of stability across this narrow range is connected to the existence of an intermediate asymmetric solution in this region, interpolating between the two solutions. When crossing the region of stability inversion, the intermediate solution appears from a pitchfork bifurcation as one of the solutions (say the 1-site solution) changes its stability and then disappears in a reversed pitchfork bifurcation with the other (2-site) solution [32]. Here, in $2 \mathrm{D}$, although the stability boundaries lie rather far apart in parameter space, unstable asymmetric intermediate solutions still exist between the stability boundaries of the 1-site solutions and both 2-site solutions (Fig. 1d) and appear as the result of similar bifurcations. Examples of the solutions can be seen in Fig. 2.

Moreover, comparing the Hamiltonians of the obtained solutions reveals the existence of zeros of the energy difference along some boundaries in parameter space. These have been indicated in Fig. 1. To better clarify the relations among the different solutions, bifurcation diagrams for $Q_{4}=0.25$ and $Q_{4}=0.1$ are shown in Figs. 3 and 4 , with Hamiltonian $\mathcal{H}$ for the solutions plotted against the parameter $Q_{5}$. Note that the solution that maximizes $\mathcal{H}$ always is stable. From Fig. 3 it is clear that the intermediate solution appears in connection with the vanishing energy difference, i.e., the difference in Hamiltonian, of the 1-site and 2-site solutions $\Delta \mathcal{H}=\mathcal{H}_{1}-\mathcal{H}_{2}$, and through bifurcations provide the mechanism of their exchange of stability. The intermediate solution always lies very close but has a slightly lower value of $\mathcal{H}$ and is therefore unstable, in contrast to the solutions in $1 \mathrm{D}$ that may be sta- 


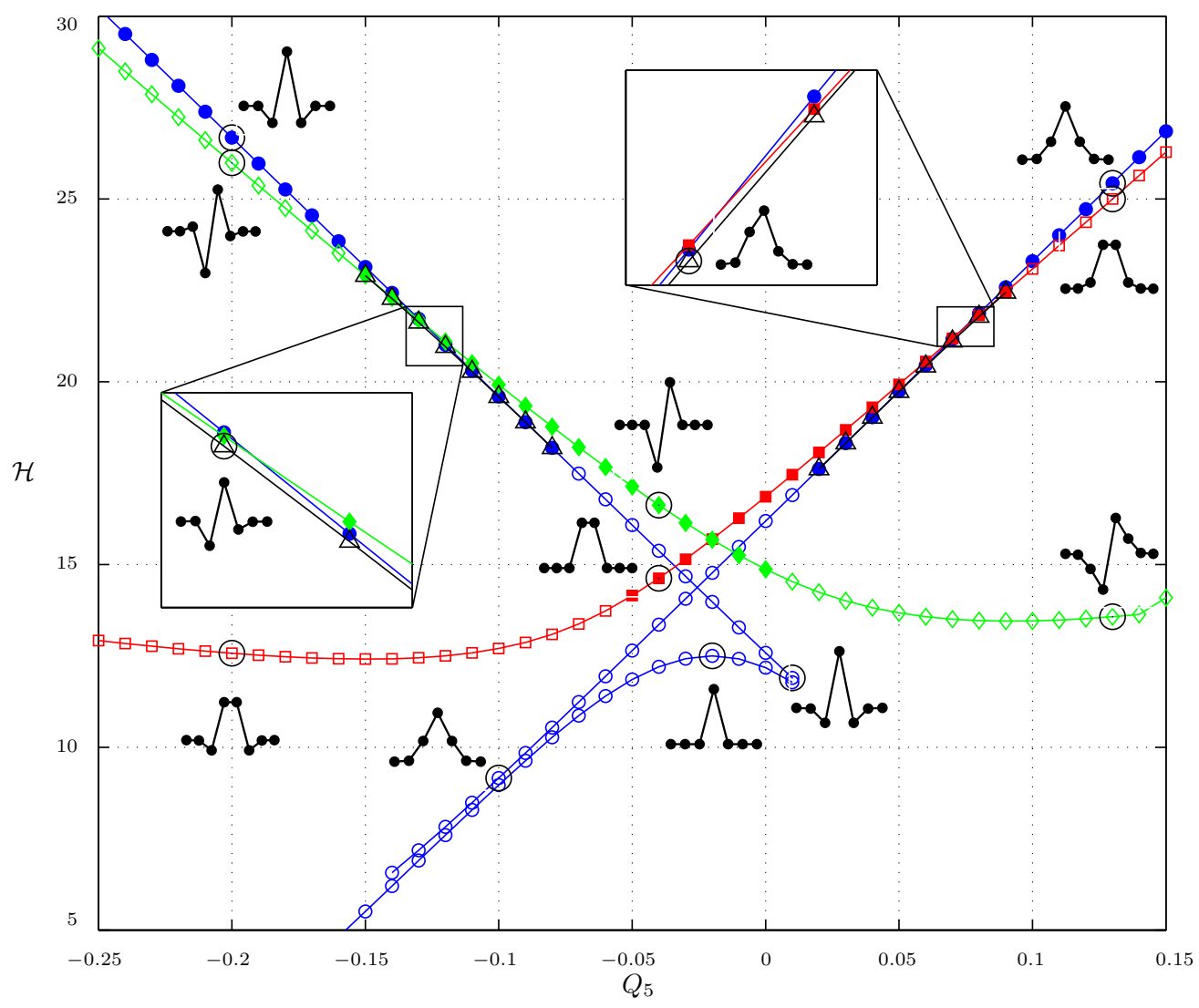

Fig. 3. (Color online) Bifurcation diagram with Hamiltonian $\mathcal{H}$ versus the parameter $Q_{5}$ for $Q_{2}=0.2, Q_{3}=0.5, Q_{4}=0.25$ and $\mathcal{N}=5$. 1-site solutions are indicated with circles (०), symmetric 2 -site solutions with squares $(\square)$, anti-symmetric 2 -site solutions with diamonds $(\diamond)$ and intermediate solutions with triangles $(\triangle)$. The markers are filled (unfilled) if the solution at that point is stable (unstable). Note also that a maximizer of the Hamiltonian always is stable. For some selected points (large circles) a one-dimensional cross section along a lattice direction of the two-dimensional solutions is shown to give a view of their characteristic form. The intermediate solutions are always unstable and exist only in a limited parameter range. They emerge from pitchfork bifurcations and connect the 1-site solution with either of the 2-site solutions. Note that they appear in connection with the vanishing of the energy difference (see insets).

ble [32]. From a mobility point of view, it is the energy of the intermediate solution that must be overcome to achieve mobility. This additional energy (the PN-barrier) is, with reference to the stable modes, small compared to the total energy $(\lesssim 1 \%)$. Note also in Fig. 3 that for each 2 -site solution there are two intersections with 1-site solutions and hence two zeros of the energy difference. One is, as expected from the energy minimum principle, present in the region of existence of the intermediate solutions, where the 1-site solution and the respective 2-site solution have simultaneous stability and are the stationary modes with highest value of $\mathcal{H}$ (see the zoomed areas). The energy 


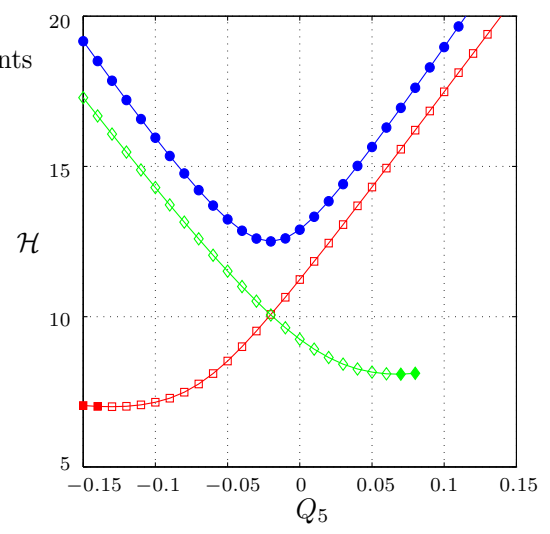

Fig. 4. (Color online) Same as in Fig. 3 but for $Q_{4}=0.1$. Note here that $\mathcal{H}$ for the 1-site solution is always larger than for the 2-site solutions.

difference is also zero in a region where the involved solutions are not the ones with highest $\mathcal{H}$, and in particular the 1-site solution is unstable in this region (see intersections at $\mathcal{H} \approx 15$ ). This intersection, as can be seen from the solution insets in Fig. 3, is with a solution possessing the 'wrong' symmetry, i.e., the nearest neighbours of the centre site have the opposite sign compared to the symmetry or anti-symmetry of the respective 2-site solution. Therefore exchange of stability, or mobility, cannot be expected at this vanishing of the energy difference.

In Fig. 3 we also see that the branches of the 1 -site solutions terminate. Two of the branches will merge at $Q_{5} \approx 0.01$, thus the end of the branches are due to bifurcations. The behaviour of the three branches is not symmetric and the two branches lying close in the left part of the figure will not coincide. Instead, the upper branch of these two is terminated at $Q_{5} \approx-0.14$ in a bifurcation with a symmetric 5-site solution that at the anti-continuous limit has 5 inphase neighbouring sites excited in a cross configuration. The lower branch can be continued to lower values of $Q_{5}$ where it will approach a plane wave solution.

\section{Mobility}

A bit surprisingly, when considering the results of the stability analysis of stationary solutions, the mobility of localized modes in the model (1) is very poor and in stark contrast to the very excellent mobility in the 1D model [32] and the saturable model in 1D [30] and 2D [31] near points of minimal PNbarrier. The method used to try to induce mobility is to apply a phase gradient $\Psi_{n, m} \mapsto \Psi_{n, m} \mathrm{e}^{\mathrm{i}\left(k_{x} n+k_{y} m\right)}$ across one of the stationary real solutions. In applications this can be achieved by launching the laser beam that excites the waveguides at an angle to the face of the array [5]. This perturbation will 


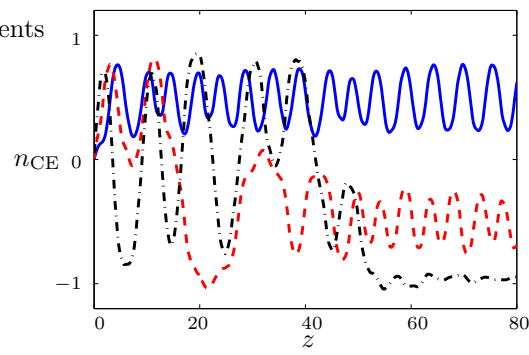

Fig. 5. (Color online) Evolution of the centre of energy when perturbing a 1-site solution for $Q_{2}=0.2, Q_{3}=0.5, Q_{4}=0.25, Q_{5}=0.05$ and $\mathcal{N}=5$ (solution shown in Fig. 2a) with a phase gradient $k_{x}$. In all cases $\mathcal{H}$ is sufficiently lowered to be below the value of the lowest stationary (the intermediate) solution with $\mathcal{H}=19.7310$. The solid line is for $k_{x}=0.1(\mathcal{H}=19.7027)$, the dotted line for $k_{x}=0.2(\mathcal{H}=19.5817)$ and the dash-dotted line for $k_{x}=0.5(\mathcal{H}=18.7839)$.

kick the solution in the direction of the phase gradient $[24,30,31,32,36]$ and will have an effect similar to the marginal mode perturbation at an instability boundary described in $[23,57]$, which in a complex formulation will correspond to a pure imaginary (velocity) perturbation to the real (position) solution. The phase gradient perturbation also has the nice feature of preserving the norm. Here we are strictly interested in axial propagation as this is the direction of possible propagation in the lattice suggested by the minimal PN-barrier of the stationary modes. Therefore $k_{y}=0$ has consistently been used. Mobility in the diagonal direction would for the nearest-neighbour coupled lattice be connected to a vanishing energy difference for the 1-site solution and a 4-site solution (quadrupole). This does not appear in the present model and the 4site solutions generally has a much lower value of $\mathcal{H}$ than the 1 -site solution. The reason that it is possible in the model in [31] is the saturable nonlinearity that will limit the amplitude of the excitations, bringing the singly excited and multiply excited solutions closer in energy for high enough power. The same effect is also the reason for the multiple zeros of the energy difference, $\Delta \mathcal{H}=\mathcal{H}_{1}-\mathcal{H}_{2}$, in saturable media, as more and more excited sites reach the saturation limit. For the model (1) we should expect at most one zero of the PN-barrier with fixed coupling parameters and varying norm $\mathcal{N}$.

Trying to kick any of the stationary solutions in the region of existence of the intermediate solution, where the PN-barrier is minimal, will result in an oscillatory behaviour near the initial position. The centre of energy for the axial direction $\hat{\mathbf{n}}$, defined as

$$
n_{\mathrm{CE}}=\frac{1}{\mathcal{N}} \sum_{n, m} n \mathcal{N}_{n, m}
$$

will at most be displaced about one lattice site. The generic behavour is examplified in Fig. 5 where the 1-site solution in Fig. 2a is perturbed with different phase gradients. Although the perturbation is large enough to over- 


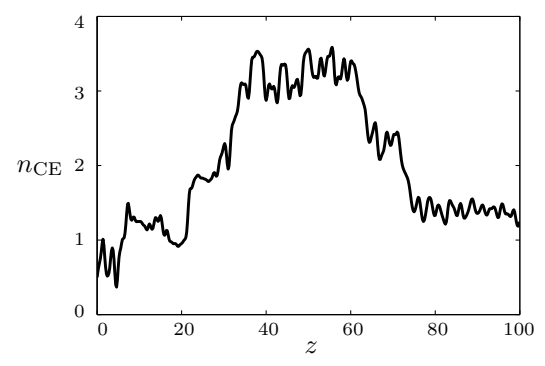

Fig. 6. Evolution of the centre of energy when perturbing an unstable symmetric 2-site solution for $Q_{2}=0.2, Q_{3}=0.5, Q_{4}=0.25, Q_{5}=-0.06$ and $\mathcal{N}=5$ with a phase gradient $k_{x}=0.2$. The motion depends sensitively on initial conditions and boundary conditions. Here periodic boundary conditions on a $21 \times 21$ lattice is used.

come the PN-barrier, set by the unstable intermediate solution, no mobility, except small displacements to neighbouring sites, is observed, indicating that the effect of pinning is stronger than suggested by the energy difference considerations. The final position of $n_{\mathrm{CE}}$ is not even always in the direction of the phase gradient kick, and is located either on or between sites corresponding to one of the stable stationary solutions. Since the initial velocity of the excitation given by the perturbation can be large, the absence of mobility here is not the effect of resonance with the multi-spectral bands appearing at low velocity for travelling waves near a zero of the PN-barrier [53,58].

If we instead start with solutions away from the region of existence of the intermediate solutions only small oscillations around the stable stationary solution in the present region is observed. Displacement of the centre of energy a few sites is however possible. In Fig. 6 an unstable symmetric 2-site solution is perturbed and the centre of energy moves up to two sites. But the irregular oscillations are due to a reconfiguration of the excitation from an initial more symmetric profile to, at the final position, a more anti-symmetric profile being closer to the stable stationary mode at the given values of the parameters. The motion is highly unpredictable and depends sensitively on the initial conditions and boundary conditions.

At the edge of the stability boundary, where the bifurcation with the intermediate solution occurs for the fundamental stationary modes, the eigenmodes related to the real eigenvalues that are responsible for the instability has a form that may be indicative of mobility. The real part of this growing mode is shown in Fig. 7 for the 1-site solution. Exactly at the stability boundary the imaginary part of the eigenmode is zero. From the form of the eigenmode we conclude that perturbing along this mode ('depinning mode' in [23]) will promote a change of shape of the 1-site solution towards a profile that resembles the symmetric 2-site solution (or the intermediate solution). The opposite relation holds at the stability boundary of the 2-site solution. Hence the form of the growing modes supports the heuristic view of mobility of narrow excita- 


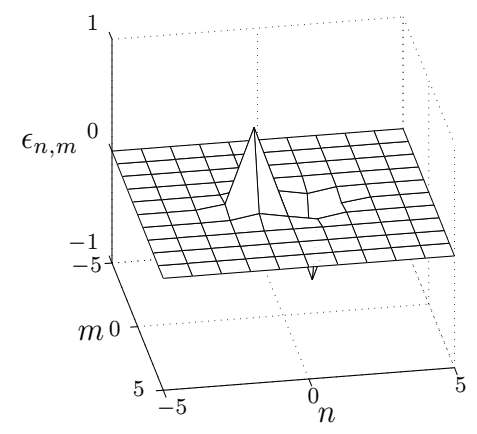

Fig. 7. Real part of the unstable eigenmode of the 1-site solution for $Q_{2}=0.2$, $Q_{3}=0.5, Q_{4}=0.25, Q_{5}=0.0165$ and $\mathcal{N}=5$. By symmetry, the eigenmode is degenerate with a corresponding eigenmode oriented along the $\hat{\mathbf{m}}$ direction. The related eigenvalue is $\lambda=0.4140$.

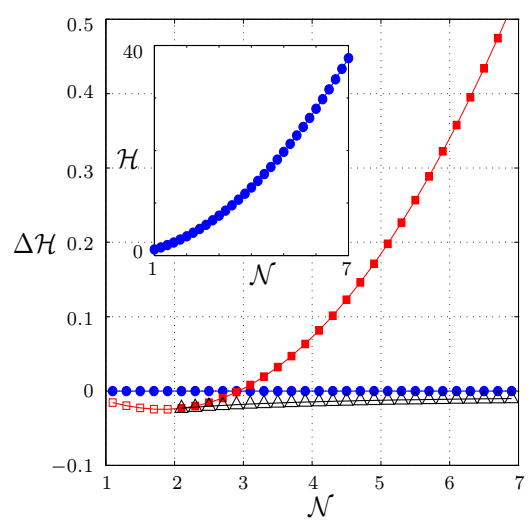

Fig. 8. (Color online) Hamiltonian $\mathcal{H}$, relative to the value of the 1 -site solution (o), versus norm $\mathcal{N}$ for $Q_{2}=0.2, Q_{3}=0.5, Q_{4}=0.25$ and $Q_{5}=0.05$. The intermediate solution $(\triangle)$ emerges from a pitchfork bifurcation with the symmetric 2 -site solution $(\square)$ at $\mathcal{N} \approx 2.1$. It is always the solution with the slightly lower value of $\mathcal{H}$ and is unstable. As $\mathcal{N}$ increases, although very close in $\mathcal{H}$, there will be no bifurcation with the 1-site solution, implying that the intermediate solution will not connect the 1-site and the 2-site solutions when considered as a function of the dynamical parameter $\mathcal{N}$. The inset shows $\mathcal{H}$ versus $\mathcal{N}$ for the 1 -site solution, as a reference.

tions as a transformation between stationary solutions (see also Fig. 3 in [32]). But in the present model the stability boundaries are far apart in parameter space and such a transformation may not be possible. To be conclusive in this matter we will need to fix the coupling parameters and investigate the exchange of stability as a function of the dynamical parameters $\mathcal{H}$ and $\mathcal{N}$. If $\mathcal{N}$ is varied for a set of fixed coupling parameters inside the right region of existence of the intermediate solution in Fig. 3 we can find a situation when the intermediate solution will only bifurcate with the symmetric 2 -site solution, and not with the 1 -site solution for reasonable values of the norm $(\mathcal{N}<100)$. This is illustrated in Fig. 8. In the event that a bifurcation occurs with both the 


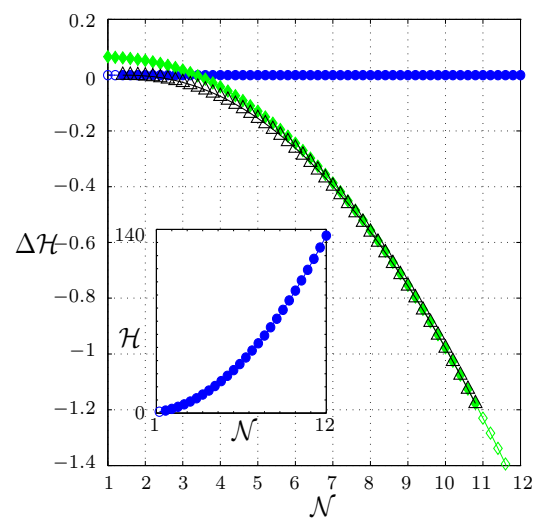

Fig. 9. (Color online) Hamiltonian $\mathcal{H}$, relative to the value of the 1-site solution (०), versus norm $\mathcal{N}$ for $Q_{2}=0.2, Q_{3}=0.5, Q_{4}=0.25$ and $Q_{5}=-0.14$. The intermediate solution $(\triangle)$ appears at $\mathcal{N} \approx 1.4$ and disappears at $\mathcal{N} \approx 10.9$ in a bifurcation with the anti-symmetric 2 -site solution $(\diamond)$. The inset shows $\mathcal{H}$ versus $\mathcal{N}$ for the 1 -site solution, as a reference.

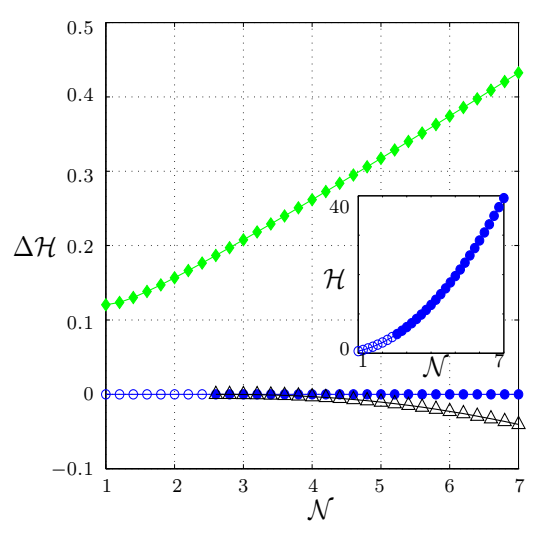

Fig. 10. (Color online) Hamiltonian $\mathcal{H}$, relative to the value of the 1-site solution (०), versus norm $\mathcal{N}$ for $Q_{2}=0.2, Q_{3}=0.5, Q_{4}=0.25$ and $Q_{5}=-0.1$. In this case the intermediate solution $(\triangle)$ emerges from a pitchfork bifurcation with the 1-site solution at $\mathcal{N} \approx 2.4$ and there is actually no zero of the energy difference. The anti-symmetric 2 -site solution $(\diamond)$ always has a higher value of $\mathcal{H}$ and is stable. The inset shows $\mathcal{H}$ versus $\mathcal{N}$ for the 1 -site solution, as a reference.

fundamental solutions involved (Fig. 9), the bifurcation points are far apart measured also in terms of the dynamical parameters. Measuring the distance between the bifurcation points in norm $(\Delta \mathcal{N})$ we find that it is always comparable in size to the value of the norm at the bifurcation point with the higher value, i.e., $\Delta \mathcal{N} / \mathcal{N} \sim 1$. The PN-barrier $(\Delta \mathcal{H})$ is small compared to $\mathcal{H}$ for all values of the norm. Since the bifurcation points are either far apart or the intermediate solution does not connect the 1-site and 2-site solutions at all, this indicates that there is no way to transform between the two. Put in another way, this suggests that there is no trajectory in phase space that passes close to the stationary configurations and consequently the mobility is very poor. 
Our numerical observations lead us to the conclusion that mobility is resisted since the site-centred and bond-centred configurations are too far apart for the adjustment of $\mathcal{N}$ and $\mathcal{H}$ to be possible by exchange with an accompanying oscillating background, or in this case mainly an adjustment of $\mathcal{N}$ since the difference in energy is always relatively small. A tractable analysis could possibly be made along the lines of the method of an effective Hamiltonian where the dynamics is averaged over periodic trajectories in phase space [59]. The more explicit interpretation of the PN-barrier as referred to a trajectory in phase space would definitely be more suitable in the present context. However, some extensions are necessary as an evaluation of the method shows that it gives an accurate description of mobility only close to limits where the travelling excitations are exact [59]. But this does not exclude that some insight might be given to the effect of pinning observed here. Further, as shown in Fig. 10, there are cases when the PN-barrier does not vanish at all, but the intermediate solution still exists.

\section{Compact solutions}

An interesting effect of the nonlinear coupling terms in Eq. (1) is that they may cancel the linear coupling and effectively give rise to a total coupling that is zero $[32,42]$. This property is also the criterion for the existence of exact compact solutions having strictly zero amplitude outside an interval [60,61]. The criterion will take the form of a parameter constraint and this implies that the compact solutions, as exact mathematical entities, are not robust with respect to parameter variation. They are interesting anyway from a physical perspective, since a near perfect localization prevails in the neigbourhood of the exact parameter values, and this family of strongly localized solutions may very well be dynamically stable. The solution will develop an exponentially decaying tail, but the contrast, i.e., the ratio of the amplitude of neighbouring sites to the amplitude of the excited sites, is still large which can be desirable for applications $[24,32]$. Further, although we speak of exact compact solutions in the context of the lattice equation (1), this does not imply compactness in the real system. The equation describes the amplitude of the modes in the respective waveguides, but the modes are themselves spatially exponentially decaying. The extreme localization represented by compact solutions will nonetheless be preferable if several excitations propagate in the system at the same time as these will constitute excitations with least mutual interaction. The existence of compact solutions has an impact on the properties of the system under study.

The idea of the compactification is that for a particular amplitude the total coupling can vanish when the neighbouring sites have zero amplitude, leading to a decoupling of the lattice between these sites. Take, e.g., the 1-site exci- 
tation with only the site $(n, m)$ excited and all others at rest and put this ansatz into Eq. (8). From the equation on one of the neighbouring sites, e.g., site $(n-1, m)$, we get $Q_{2}+2 Q_{5}\left|\psi_{n, m}\right|^{2}=0$. For a compact solution with $M$ excited sites, this constraint generalises to

$$
Q_{2}+2 Q_{5} \frac{\mathcal{N}}{M}=0
$$

if all sites have the same amplitude. Note that $Q_{5}<0$ is required for the existence of compact solutions (as $Q_{2}>0$ ). This criterion corresponds to having a negative Kerr index of the nonlinear material surrounding the waveguides, or for BEC in an optical lattice an effective interatomic attraction in the dilute gas (as for ${ }^{7} \mathrm{Li}$ ). Solving for the 1-site and 2-site compact solutions the results are the same as in 1D $[32,42]$. From Eq. (10) the constraint for the 1-site solution will correspond to the line $Q_{5}=-0.02$ in Fig. 1, that precisely cuts through the edge of the instability region of the 1-site solution and hence also seperates the two different regions of existence of the intermediate solutions. The loose interpretation of this constraint as an effective coupling is further strenghtened when considering that the two 2-site solutions will have the same value of $\mathcal{H}$ along the plane of existence of the 1 -site solution. Note, e.g., that the lines of $\mathcal{H}$ intersect at $Q_{5}=-0.02$ in the bifurcation diagrams in Figs. 3 and 4 .

Compact solutions with more than two sites excited can also be found. There are two different classes of real 3-site solutions, with the center site being either in-phase or out-of-phase with the two outermost sites. Having, in 2D, the freedom to choose the orientation of the three sites in the lattice as being all along one lattice direction or in an 'L'-configuration, the presence of coupling in two different lattice directions will also mean less freedom when compared to the $1 \mathrm{D}$ system. The extra restrictions imposed for the 3 -site solutions implies that all sites must have the same amplitude, which is not required in 1D. Explicit calculations yield the same frequency $\Lambda=2 Q_{3} \mathcal{N} / 3$ for all solutions, and apart from the parameter constraint (10) also the constraint $Q_{4}=\mp Q_{5}$, where the upper sign is for the in-phase solution and the lower sign for the out-of-phase solution. A check of the stability shows that only the out-of-phase solution will be stable for $\mathcal{N} \geq 8.72$, regardless of the orientation in the lattice. It is also possible to solve analytically for compact 4-site and 5-site solutions, some of which are stable for large enough values of the norm $(\mathcal{N} \gtrsim 20)$.

\subsection{Vortices and complex solutions}

An interesting extension when going from one to two spatial dimensions is the possiblity to support localized excitations having a topological charge, which is proportional to the angular momentum carried by the excitation. 

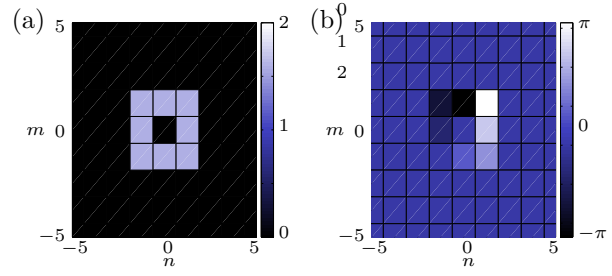

Fig. 11. (Color online) (a) The amplitude $\sqrt{\mathcal{N}_{n, m}}=\left|\Psi_{n, m}\right|$ and (b) the phase $\theta_{n, m}=\arg \left\{\Psi_{n, m}\right\}$ for a stable stationary compact vortex of topological charge $S=1$ for $\mathcal{N}=20, Q_{2}=0.2, Q_{3}=0.5, Q_{4}=0.04$ and $Q_{5}=-4 Q_{2} / \mathcal{N}=-0.04$. The frequency is given by $\Lambda=-3 \sqrt{2} Q_{2}+Q_{3} \mathcal{N} / 4+Q_{4} \mathcal{N}=2.4515$.

We define the topological charge $S$ as the number of complete $2 \pi$ twists of the phase when moving on a contour around the excitation. Such discrete vortex solitons have been observed in experiments [10,11] and described in the context of DNLS-type equations $[62,63,64,65]$. In the model (1) compact vortices can be supported. The constraint (10) must still be fulfilled, which means, as we have fixed $Q_{2}$ and $Q_{3}$ by rescalings, that the parameter $Q_{5}$ is fixed if the norm $\mathcal{N}$ and $Q_{4}$ are used as free parameters. The most simple compact vortex is given by four excited sites in a square configuration with a phase difference of $\pi / 2$ between neighbouring sites giving a topological charge $S=1$. This solution will have the frequency $\Lambda=Q_{3} \mathcal{N} / 2+Q_{4} \mathcal{N}$ and have a very small region of stability near $Q_{4}=0$ (e.g., for $\mathcal{N}=10$ it is stable for $\left.Q_{4} \leq 0.0023\right)$. For the square 4-site configuration placed diagonally in the lattice, with a zero-amplitude mediating site in the middle, the frequency is instead $\Lambda=Q_{3} \mathcal{N} / 2$ and the region of stability is dramatically extended so that for $\mathcal{N}=10$ the configuration is stable for $Q_{4} \leq 0.1634$. The excited sites are not nearest neighbours in this case and will only weakly interact. In fact for $\mathcal{N}=20$ the instability will set in at $Q_{4}=0.165$, which exactly corresponds to the instability of the compact 1 -site solution for $\mathcal{N}=5$. With more excited sites it is possible to find larger $S=1$ vortices that are stable for certain parameter values. In Fig. 11 an example with eight sites is shown. Also higher order compact vortices exist. The configuration with eight sites on a square contour with phase difference $\pi / 2$ between the sites will have charge $S=2$ and frequency $\Lambda=Q_{3} \mathcal{N} / 4+Q_{4} \mathcal{N} / 2$. It will also be stable for large norm $(\mathcal{N} \gtrsim 20)$ and small $Q_{4}$. (Note that, by contrast, this $S=2$ configuration is always unstable in the standard on-site DNLS model [64].)

The vortex solutions are necessarily complex solutions as they have a phase difference between neighbouring sites other than 0 or $\pi$, i.e., they cannot be transformed to real form using the global phase invariance of the DNLS-type equations. This implies that the norm current, Eq. (7), is non-zero and that there is a flow of norm around the contour of the vortex balanced according to the discrete continuity equation (6) with constant norm density $\mathcal{N}_{n, m}$. As described for the 1D model in [42] the presence of the nonlinear coupling will introduce a non-trivial phase dependence in the norm current density. 
In particular, the norm current $\mathcal{J}_{n, m}^{(n)}$ in Eq. (7) can become zero for specific values of the phase difference given by

$$
\cos \varphi_{n, m}=-\frac{Q_{2}+2 Q_{5}\left(\mathcal{N}_{n, m}+\mathcal{N}_{n+1, m}\right)}{4 Q_{4} \sqrt{\mathcal{N}_{n, m} \mathcal{N}_{n+1, m}}}
$$

Thus under the constraint (11) stationary complex solutions can exist without having to form a closed loop for the norm current flow, which is the case for a vortex, simply because there is no current. The compact complex 2site solution present in the $1 \mathrm{D}$ model [42] exists also here, with $\cos \varphi_{n, m}=$ $Q_{2} / 2 Q_{4} \mathcal{N}$ and $\Lambda=Q_{3} \mathcal{N}+Q_{4} \mathcal{N}-3 Q_{2}^{2} / 2 Q_{4} \mathcal{N}$. It is also stable for a range of phase twists, e.g., for $\mathcal{N}=10$ stability is found for $0.010 \leq Q_{4} \leq 0.0420$, equivalent to $0.2381 \leq \cos \varphi_{n, m} \leq 1$. Also compact complex solutions with more sites excited can be constructed, e.g., 4-site non-vortex solutions that generally seem to be unstable. Neither these solutions nor the vortex solutions above need to be compact, but can be continued to non-compact solutions by lifting the constraint set by Eq. (10). Although this may be an interesting further development the given examples serve to give an idea of the phenomena at hand.

\section{Power transport by plane waves}

Leaving the localized solutions, we will in this section focus on the transport properties of plane waves. The excitation of the lattice with a plane wave correponds to an even distribution of the energy over all sites in the lattice. A travelling wave on the form

$$
\Psi_{n, m}(z)=\sqrt{\rho} \mathrm{e}^{-\mathrm{i}(\varphi n+\phi m+\Lambda z)}
$$

will have a norm density (power per site) $\mathcal{N}_{n, m} \equiv \rho$ and be a solution of Eq. (1) for the frequency

$$
\begin{aligned}
\Lambda= & 2 Q_{3} \rho+2\left(Q_{2}+8 Q_{5} \rho\right)(\cos \varphi+\cos \phi) \\
& +4 Q_{4} \rho(4+\cos 2 \varphi+\cos 2 \phi) .
\end{aligned}
$$

Note that the dispersion relation (13) can be derived from Eq. (5a) in the form $\Lambda=\partial \mathcal{H}_{n, m} / \partial \rho$. Though being a stationary solution, the plane wave (12) is associated with a balanced transfer of norm (power) between the sites due to the induced phase difference (wave number). This balance is described by the discrete continuity equation (6) with constant norm density. From the interpretation of $\mathcal{J}^{(n)} \equiv \mathcal{J}_{n, m}^{(n)}$ and $\mathcal{J}^{(m)} \equiv \mathcal{J}_{n, m}^{(m)}$ as the norm transferred from a given site to its neighbours in the positive $\hat{\mathbf{n}}$ and $\hat{\mathbf{m}}$ directions we can, from Eq. (7), obtain the norm current flowing in the lattice for a plane wave 
solution,

$$
\begin{aligned}
\mathcal{J}= & \mathcal{J}^{(n)} \hat{\mathbf{n}}+\mathcal{J}^{(m)} \hat{\mathbf{m}}=-\frac{\partial \mathcal{H}_{n, m}}{\partial \varphi} \hat{\mathbf{n}}-\frac{\partial \mathcal{H}_{n, m}}{\partial \phi} \hat{\mathbf{m}} \\
= & 2 \rho \sin \varphi\left[Q_{2}+4 Q_{4} \rho \cos \varphi+4 Q_{5} \rho\right] \hat{\mathbf{n}} \\
& +2 \rho \sin \phi\left[Q_{2}+4 Q_{4} \rho \cos \phi+4 Q_{5} \rho\right] \hat{\mathbf{m}} .
\end{aligned}
$$

Further, it is convenient to introduce a polar representation of the current defined by the relation $|\mathcal{J}| \mathrm{e}^{\mathrm{i} \alpha}=\mathcal{J}^{(n)}+\mathrm{i} \mathcal{J}^{(m)}$, so that

$$
|\mathcal{J}|=\sqrt{\mathcal{J}^{(n) 2}+\mathcal{J}^{(m) 2}}
$$

is the magnitude of the current and

$$
\left.\alpha=\arctan \left(\frac{\mathcal{J}^{(m)}}{\mathcal{J}^{(n)}}\right)( \pm \pi) \quad \in\right]-\pi, \pi[
$$

its direction measured counter clockwise relative to the $\hat{\mathbf{n}}$ direction. Note that the power transported along the waveguides is proportional to $\rho$ and that the quantities (14), (15) and (16) describe the transport across the array. For a finite array such transport can only be sustained if there is a supply of power at one boundary and a drain at the opposite boundary.

\subsection{Modulational stability}

To ensure that the plane wave (12) has stable propagation its behaviour under a modulation is investigated. Adding a modulating perturbation with wave numbers $q$ and $p$ we make the ansatz

$$
\begin{aligned}
\Psi_{n, m}(z)= & {\left[\sqrt{\rho}+u(z) \mathrm{e}^{\mathrm{i}(q n+p m)}+v^{*}(z) \mathrm{e}^{-\mathrm{i}(q n+p m)}\right] } \\
& \times \exp [-\mathrm{i}(\varphi n+\phi m+\Lambda z)]
\end{aligned}
$$

in Eq. (1) $[42,43,44,45,66]$. Keeping only terms linear in $u$ and $v$, the modulational stability can be determined from the equations

$$
\mathrm{i} \frac{d}{d z}\left(\begin{array}{l}
u \\
v
\end{array}\right)=\left(\begin{array}{cc}
a+b & c \\
-c & a-b
\end{array}\right)\left(\begin{array}{l}
u \\
v
\end{array}\right)=\omega_{ \pm}\left(\begin{array}{l}
u \\
v
\end{array}\right)
$$


with

$$
\begin{aligned}
a= & 2\left(Q_{2}+8 Q_{4} \rho \cos \varphi+8 Q_{5} \rho\right) \sin \varphi \sin q \\
& +2\left(Q_{2}+8 Q_{4} \rho \cos \phi+8 Q_{5} \rho\right) \sin \phi \sin p, \\
b= & 2 Q_{3} \rho-2 Q_{2}(\cos \varphi+\cos \phi) \\
& +4 Q_{4} \rho[2 \cos q(1+\cos 2 \varphi)-\cos 2 \varphi] \\
& +4 Q_{4} \rho[2 \cos p(1+\cos 2 \phi)-\cos 2 \phi] \\
& +2\left(Q_{2}+8 Q_{5} \rho\right)(\cos \varphi \cos q+\cos \phi \cos p), \\
c= & 2 Q_{3} \rho+4 Q_{4} \rho(\cos 2 \varphi+2 \cos q+\cos 2 \phi+2 \cos p) \\
& +8 Q_{5} \rho[\cos \varphi(1+\cos q)+\cos \phi(1+\cos p)] .
\end{aligned}
$$

The plane wave will be stable if the eigenfrequencies $\omega_{ \pm}=a \pm \sqrt{b^{2}-c^{2}}$ are real for all $q, p \in]-\pi, \pi]$. By explicit calculation we find that the eigenfrequencies can be written on the form

$$
\begin{aligned}
\omega_{ \pm}= & \frac{\partial \mathcal{J}^{(n)}}{\partial \rho} \sin q+\frac{\partial \mathcal{J}^{(m)}}{\partial \rho} \sin p \\
\pm & {\left[\left(\frac{4 \sin ^{2} \frac{q}{2}}{m_{\mathcal{H}}^{(n)}(\varphi)}+\frac{4 \sin ^{2} \frac{p}{2}}{m_{\mathcal{H}}^{(m)}(\phi)}\right)\right.} \\
& \left.\times\left(f(\varphi) \sin ^{2} \frac{q}{2}+f(\phi) \sin ^{2} \frac{p}{2}+\rho \frac{\partial \Lambda}{\partial \rho}\right)\right]^{1 / 2}
\end{aligned}
$$

where we have introduced the (energetic) effective mass

$$
\begin{aligned}
\frac{1}{m_{\mathcal{H}}^{(n)}} & =\frac{1}{\rho} \frac{\partial^{2} \mathcal{H}_{n, m}}{\partial \varphi^{2}}=-\frac{1}{\rho} \frac{\partial \mathcal{J}^{(n)}}{\partial \varphi} \\
& =-2\left(Q_{2}+4 Q_{5} \rho\right) \cos \varphi-8 Q_{4} \rho \cos 2 \varphi
\end{aligned}
$$

with a similar expression for $m_{\mathcal{H}}^{(m)}(\phi)$, and the function

$$
f(\varphi)=-2\left[\left(Q_{2}+12 Q_{5} \rho\right) \cos \varphi+4 Q_{4} \rho\left(2 \cos ^{2} \varphi+1\right)\right]
$$

entirely in correspondence with the result in 1D [42]. From the first factor in Eq. (20) we see that if the effective masses have opposite signs there will be at least some values of $q$ and $p$ that will yield instability. An important observation that follows is that if $1 / m_{\mathcal{H}}^{(n)}$ or $1 / m_{\mathcal{H}}^{(m)}$ change sign due to tuning of some available parameters, the plane wave can only change its stability or remain unstable (unless the second factor in Eq. (20) changes sign at the same time). 


\subsection{Power currents}

Generally, the direction of power flow in a lattice will be completely determined by the induced phase gradient of the plane wave. For the cubic on-site DNLS equation $\left(Q_{4}=Q_{5}=0\right)$, e.g., the direction of the norm current is fixed by the relative phases between the sites, $\tan \alpha \propto \sin \phi / \sin \varphi$, while the magnitude is linear in the amplitude, $|\mathcal{J}| \propto \rho$. Thus any control of the norm current by tunable parameters is restricted to the wave numbers of the plane wave. With current experimental techniques, a phase gradient is induced by having the exciting laser beam directed at an angle to the array of waveguides [5], so the wave numbers are easily adjustable but not rapidly tuned. Also, for a finite array the wave numbers will be quantized in order to satisfy the imposed boundary conditions.

As discussed in [42], with the introduction of nonlinear coupling terms in the model (1), the norm current will have a nonlinear dependence on the amplitude. This will make $\rho$ more suitable as a tunable parameter, also from an application point of view. Including the $Q_{5}$ term, but keeping $Q_{4}=0$ (which would be relevant for application to BEC in an optical potential $[43,44,45])$, will introduce a nonlinear dependence in the magnitude $|\mathcal{J}|$ through the factor $Q_{2}+4 Q_{5} \rho$. The sign of this factor will determine in which of the two possible directions $(\alpha)$, differing by an angle $\pi$ and fixed by the phase gradients $\varphi$ and $\phi$, the current will flow. For $Q_{5}<0$ it is hence possible to make the current zero for a finite value of the amplitude $\rho$ and at the same time reverse its direction, just as in $1 \mathrm{D}$ [42]. However, as the factor $Q_{2}+4 Q_{5} \rho$ appears also in the effective mass (21), the change of sign will affect the stability of the travelling plane wave. In particular, as noted in Sec. 6.1, the plane wave cannot be modulationally stable for both directions of the current near the point of inversion.

Inclusion also of the $Q_{4}$ term will change this situation, as the zeros of $\mathcal{J}^{(n)}$ and $1 / m_{\mathcal{H}}^{(n)} \propto \partial \mathcal{J}^{(n)} / \partial \varphi$ in general no longer will coincide. However, numerical investigations reveal that stable inversion is not possible in the axial direction, i.e., with $\phi=0, \pi$, for any values of $\varphi$ and $Q_{4}>0$, but it is possible in other directions. Taking $\varphi=\phi \neq 0, \pi$ will give a diagonal direction of the current, with $\alpha=\pi / 4,-3 \pi / 4$ depending on the relative signs of $\mathcal{J}^{(n)}$ and $\mathcal{J}^{(m)}$, and always stability near the point of inversion. An example of such a flip of direction as the amplitude of the plane wave is varied is shown in Fig. 12. An even greater flexibility is presented if different phase gradients are chosen for the $\hat{\mathbf{n}}$ and $\hat{\mathbf{m}}$ directions, $\varphi \neq \phi$. As illustrated in Fig. 12 we may have the remarkable situation that as the amplitude $\rho$ is tuned the magnitude of the current is near constant while the direction is continuously changed an angle $\pi / 2$ over a stable range. Such an exact control of the power flow by simply changing the amplitude of the plane wave may find useful applications. One 

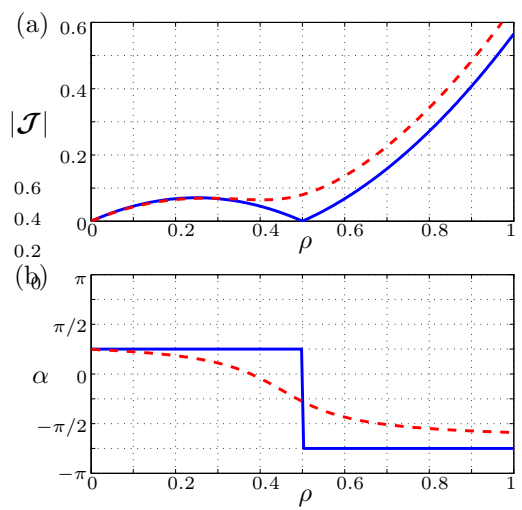

Fig. 12. (Color online) (a) The magnitude $|\mathcal{J}|$ and (b) the direction $\alpha$ of the norm current for varying amplitude $\rho$ with the parameters $Q_{2}=0.2, Q_{3}=0.5, Q_{4}=0.1$ and $Q_{5}=-0.1$. The solid line is for $\varphi=\phi=\pi / 2$, and the plane wave is stable for all $\rho$. The dashed line is for $\varphi=1.3$ and $\phi=1.9$, and the plane wave is stable for $\rho>0.119$.

possible application could be to use the array of waveguides, excited with a plane wave, as the link in a power coupling device. The advantage over a direct coupling would be a greater control of the flow facilitated by the control of the excitation in the waveguides. The effect of current inversion could form the basis for an amplitude-operated power-switch. However, any discussions in this direction other than mere speculative are beyond the scope of this paper.

\section{Conclusion}

In conclusion, we have studied the extension to two spatial dimensions of a one-dimensional model describing coupled optical waveguides going beyond the lowest-order approximation and including nonlinear coupling, which, e.g., is relevant for arrays of linear waveguides embedded in nonlinear media. The phenomenology of the 1D model presented in a series of papers $[32,42,56]$ has been revisited in the 2D setting. Especially, we have shown that a vanishing energy difference between 1-site and 2-site stationary solutions and a subsequent small Peierls-Nabarro energy barrier, taking into account also the existence of asymmetric intermediate solutions, does not result in good mobility of highly localized modes. The main reason, and the difference to models that show good mobility $[30,31,32]$, is that, despite small energy differences, the stationary solutions are still in some sense far apart. Rather, the bifurcation points where the fundamental modes change their stability and where they exhibit a depinning mode promoting mobility are, although close in Hamiltonian $(\mathcal{H})$, far apart in norm $(\mathcal{N})$ (Figs. 8 and 9). From this we conclude that for the stationary solutions that have near equal values of both Hamiltonian and norm there is likely no trajectory in phase space passing close to them both. Thus, in 
order to have good mobility of narrow modes in discrete systems, we need not only a small PN-barrier but also an exchange of stability between 1-site and 2 -site solutions taking place in the vicinity. We therefore also conjecture that the size of the oscillating background accompanying travelling modes $[27,28]$ is not only related to the size of the PN-barrier but also to the difference in other conserved quantities (like $\mathcal{N}$ ) of the involved stationary modes measured at the points where their stability is changed. The lack of mobility near a zero of the energy difference between fundamental modes was observed also in [36], but no further analysis was carried out.

Effects of a more delicate balance between linear and nonlinear coupling terms in Eq. (1) have also been discussed. These include the existence and stability of compact solutions, both discrete breathers and discrete vortex solitons. The latter have to our knowledge not been previously reported. Mathematically, these solutions are not robust with respect to parameter variation and require a balance of the coupling parameters in the equation for exact compactness, but near perfect localization persists in the neighborhood of the exact parameter values. An interesting question is if they can prevail also beyond the tight-binding approximation of Eq. (1). The answer is likely that they are only exact solutions for the present model, but that they will correspond to modes of a higher degree of localization in more accurate models, i.e., they give an indication in which parameter regimes high localization can be achieved. As discussed in Sec. 5 the compact solutions do not represent a true compactification in the real system.

Additional interesting effects present also in 1D arise from a non-trivial dependence of the power flow in the lattice on the amplitude and phase difference of an excitation. Particularly, the current can become zero for a non-trivial phase twist which also in 2D leads to the existence of complex localized stationary modes with an open geometry, that may even be stable. Further, we have shown how the transversal flow of power in the array of waveguides can be controlled with great flexibility by excitation with plane waves, and in particular explicitly demonstrated how the transport direction may be continuously tuned by amplitude variation. This may have applications for power-coupling devices. However, the use of a 2D array of the type studied here for multiport switching is discouraged due to the poor mobility of localized modes. For this purpose a 1D array [32] or an array exhibiting a saturable [31] or quadratic [67] (see discussion below) nonlinearity instead of the Kerr nonlinearity is better suited.

We end by commenting briefly on a number of works on related issues, that appeared in print after the original submission of our manuscript.

In [68], Abdullaev et.al extended the Wannier-function approach of [18] for the continuous 1D NLS equation with periodic linear potential to the case 
with periodic variation also for the nonlinearity coefficient (which is the case also considered in our work). Within the tight-binding approximation, they derived under quite general conditions a lattice equation, which in a special case $(\sigma=1$ in [68]) is equivalent to the $1 \mathrm{D}$ version of our Eq. (1) as derived in [32]. An important conclusion of [68] is the crucial importance to include also the nonlinear coupling terms (corresponding to our $Q_{4}$ and $Q_{5}$ ), as they for specific choices of nonlinear interactions were shown to be comparable with, or even stronger than, the on-site nonlinearity. Thus, this supports the soundness of our approach to consider variation of the parameters $Q_{4}$ and $Q_{5}$ over a rather large range of values.

Several works discussing properties of moving solitons and (vanishing) PNbarriers in 1D generalized DNLS models have appeared. Dmitriev, Khare et al. $[69,70]$ found analytically exact stationary and moving solutions for some of the exceptional, translationally invariant discretizations of $[33,34,35]$. Whether these are of relevance for any physically realizable system is, to our knowledge, still unclear. Pelinovsky et al. [71] developed a mathematical technique for analysis of persistence of traveling single-humped localized solutions from a certain limit, and found specifically that while travelling solutions terminated when continued from the integrable AL-limit of the Salerno model (corresponding to the development of a resonant tail [27]), they generally persisted in the translationally invariant models. Oxtoby and Barashenkov [72] used the method of asymptotics beyond all orders to evaluate the amplitude of radiation from a moving small-amplitude soliton in the saturable DNLS equation, and found it to be completely suppressed at certain 'sliding velocities', where, similarly as in [53], it was interpreted as an embedded soliton. The properties of the travelling solitary waves in the saturable model were also analyzed numerically in more detail by Melvin et al. in [73].

For the two-dimensional case, Susanto et al. [67] studied the mobility of discrete solitons in a square lattice with quadratic (second-harmonic-generating) nonlinearity. In this case, due to the absence of collapse instability in the continuum limit, good mobility of stable solutions was observed as long as the coupling constants were not too small ('quasicontinuum regime'). In this regime, mobility in arbitrary directions (not necessarily axial or diagonal) was observed, which is not surprising since discreteness effects are smoothened out for broad, continuum-like solitons. However, no non-trivial zeros of the PN barrier were found for this model, and consequently strongly localized solutions were reported to be immobile. Thus, in many aspects, the mobility properties of the $2 \mathrm{D}$ model with quadratic nonlinearity is similar to those of the 1D cubic on-site DNLS model.

Finally, a very recent preprint by Chong et al. [74] extended the analysis of the on-site cubic-quintic DNLS model of [38] to higher dimensions. As concerns the two-dimensional mobility, the results were shown to be very similar 
to that of the saturable model [31]: enhanced mobility was found in regimes close to stability inversion and associated with appearance of asymmetric intermediate solutions and low PN barrier. Thus, as far as we are aware, the present work still provides the only known explicit example of a model where these properties do not lead to an enhanced mobility.

\section{Acknowledgements}

MÖ would like to thank the Mathematics Department at Heriot-Watt University, Edinburgh, for its hospitality and especially J.C. Eilbeck for guidance, support and very useful discussions. This work was partly carried out under the HPC-EUROPA project (RII3-CT-2003-506079), with the support of the European Community - Research Infrastructure Action under the FP6 "Structuring the European Research Area" Programme. Partial support from the Swedish Research Council is acknowledged.

\section{References}

[1] Yu.S. Kivshar and G.P. Agrawal, Optical Solitons: From Fibers to Photonic Crystals (Academic Press, San Diego, 2003).

[2] D.N. Christodoulides, F. Lederer and Y. Silberberg, Nature 424, 817 (2003).

[3] H.S. Eisenberg, Y. Silberberg, R. Morandotti, A.R. Boyd and J.S. Aitchison, Phys. Rev. Lett. 81, 3383 (1998).

[4] R. Morandotti, U. Peschel, J.S. Aitchison, H.S. Eisenberg and Y. Silberberg, Phys. Rev. Lett. 83, 2726 (1999).

[5] R. Morandotti, H.S. Eisenberg, Y. Silberberg, M. Sorel and J.S. Aitchison, Phys. Rev. Lett. 86, 3296 (2001).

[6] T. Pertsch, U. Peschel, J. Kobelke, K. Schuster, H. Bartelt, S. Nolte, A. Tünnermann and F. Lederer, Phys. Rev. Lett. 93, 053901 (2004).

[7] F. Chen, M. Stepić, C.E. Rüter, D. Runde, D. Kip, V. Shandarov, O. Manela and M. Segev, Opt. Express 13, 4314 (2005).

[8] J.W. Fleischer, T. Carmon, M. Segev, N.K. Efremidis and D.N. Christodoulides, Phys. Rev. Lett. 90, 023902 (2003).

[9] J.W. Fleischer, M. Segev, N.K. Efremidis and D.N. Christodoulides, Nature 422, 147 (2003).

[10] D.N. Neshev, T.J. Alexander, E.A. Ostrovskaya, Yu.S. Kivshar, H. Martin, I. Makasyuk and Z. Chen, Phys. Rev. Lett. 92, 123903 (2004). 
[11] J.W. Fleischer, G. Bartal, O. Cohen, O. Manela, M. Segev, J. Hudock and D.N. Christodoulides, Phys. Rev. Lett. 92, 123904 (2004).

[12] S.M. Jensen, IEEE J. Quantum Electron. 18, 1580 (1982).

[13] D.N. Christodoulides and R.I. Joseph, Opt. Lett. 13, 794 (1988).

[14] M. Stepić, D. Kip, Lj. Hadžievski and A. Maluckov, Phys. Rev. E 69, 066618 (2004).

[15] N.K. Efremidis, S. Sears, D.N. Christodoulides, J.W. Fleischer and M. Segev, Phys. Rev. E 66, 046602 (2002).

[16] S.F. Mingaleev and Yu.S. Kivshar, Phys. Rev. Lett. 86, 5474 (2001).

[17] A. Yariv, IEEE J. Quantum Electron. 9, 919 (1973).

[18] G.L. Alfimov, P.G. Kevrekidis, V.V. Konotop and M. Salerno, Phys. Rev. E 66, 046608 (2002).

[19] A. Scott, Nonlinear Science - Emergence 83 Dynamics of Coherent Structures (Oxford University Press, Oxford, 1999).

[20] D.K. Campbell, S. Flach and Yu.S. Kivshar, Phys. Today 57(1), 43 (2004).

[21] Ch. Claude, Yu.S. Kivshar, O. Kluth and K.H. Spatschek, Phys. Rev. B 47, 228 (1993).

[22] Yu.S. Kivshar and D.K. Campbell, Phys. Rev. E 48, 3077 (1993).

[23] D. Chen, S. Aubry and G.P. Tsironis, Phys. Rev. Lett. 77, 4776 (1996).

[24] O. Bang and P.D. Miller, Phys. Scr. T 67, 26 (1996).

[25] J. Juul Rasmussen and K. Rypdal, Phys. Scr. 33, 481 (1986).

[26] M.J. Ablowitz and J.F. Ladik, J. Math. Phys. 16, 598 (1975); ibid. 17, 1011 (1976).

[27] J. Gómez-Gardeñas, F. Falo and L.M. Floría, Phys. Lett. A 332, 213 (2004).

[28] J. Gómez-Gardeñas, L.M. Floría and A.R. Bishop, Physica D 216, 31 (2006).

[29] A.V. Savin, Y. Zolotaryuk and J.C. Eilbeck, Physica D 138, 267 (2000).

[30] Lj. Hadžievski, A. Maluckov, M. Stepić and D. Kip, Phys. Rev. Lett. 93, 033901 (2004).

[31] R.A. Vicencio and M. Johansson, Phys. Rev. E 73, 046602 (2006).

[32] M. Öster, M. Johansson and A. Eriksson, Phys. Rev. E 67, 056606 (2003).

[33] S.V. Dmitriev, P.G. Kevrekidis, A.A. Sukhorukov, N. Yoshikawa and S. Takeno, Phys. Lett. A 356, 324 (2006).

[34] D.E. Pelinovsky, Nonlinearity 19, 2695 (2006). 
[35] P.G. Kevrekidis, S.V. Dmitriev and A.A. Sukhorukov, Mathematics and Computers in Simulation 74, 343 (2007).

[36] A. Maluckov, M. Stepić, D. Kip and Lj. Hadžievski, Eur. Phys. J. B 45, 539 (2005).

[37] A. Maluckov, Lj. Hadžievski and M. Stepić, Physica D 216, 95 (2006).

[38] R. Carretero-Gonźalez, J.D. Talley, C. Chong and B.A. Malomed, Physica D 216, 77 (2006).

[39] T. Cretegny, Ph.D. thesis (in French), École Normale Supérieure de Lyon (1998).

[40] S. Aubry, Physica D 216, 1 (2006).

[41] A. Khare, K.Ø. Rasmussen, M.R. Samuelsen and A. Saxena, J. Phys. A: Math. Gen. 38, 807 (2005).

[42] M. Öster and M. Johansson, Phys. Rev. E 71, 025601(R) (2005).

[43] A. Smerzi and A. Trombettoni, Chaos 13, 766 (2003).

[44] A. Smerzi and A. Trombettoni, Phys. Rev. A 68, 023613 (2003).

[45] C. Menotti, A. Smerzi and A. Trombettoni, New J. Phys. 5, 112 (2003).

[46] M. Öster, Stability and Mobility of Localized and Extended Excitations in Nonlinear Schrödinger Models, Doctoral thesis, Linköping Studies in Science and Technology. Dissertations, No. 1072, ISBN 978-91-85715-83-1, Linköping University (2007); published electronically at http://urn.kb.se/resolve?urn=urn:nbn:se:liu:diva-8091.

[47] H. Goldstein, Classical Mechanics, 2nd ed. (Addison-Wesley, Massachusetts, 1980).

[48] M.I. Weinstein, Nonlinearity 12, 673 (1999).

[49] R.S. MacKay and S. Aubry, Nonlinearity 7, 1623 (1994).

[50] J.-A. Sepulchre and R.S. MacKay, Nonlinearity 10, 679 (1997).

[51] J.C. Eilbeck, P.S. Lomdahl, and A.C. Scott, Phys. Rev. B 30, 4703 (1984).

[52] J.L. Marín and S. Aubry, Nonlinearity 9, 1501 (1996).

[53] T.R.O. Melvin, A.R. Champneys, P.G. Kevrekidis and J. Cuevas, Phys. Rev. Lett. 97, 124101 (2006).

[54] J. Carr and J.C. Eilbeck, Phys. Lett. 109A, 201 (1985).

[55] C. Robinson, Dynamical Systems: Stability, Symbolic Dynamics and Chaos, 2nd ed. (CRC Press, Boca Raton, 1999).

[56] M. Öster and M. Johansson, in Proceedings of the Conference on Localization and Energy Transfer in Nonlinear Systems, San Lorenzo de Escorial, Madrid, Spain, 2002, edited by L. Vázquez, M.P. Zorzano and R.S. MacKay (World Scientific, Singapore, 2003). 
[57] S. Aubry and T. Cretegny, Physica D 119, 34 (1998).

[58] A.A. Aigner, A.R. Champneys and V.M. Rothos, Physica D 186, 148 (2003).

[59] R.S. MacKay and J.-A. Sepulchre, J. Phys. A: Math. Gen. 35, 3985 (2002).

[60] P.G. Kevrekidis and V.V. Konotop, Phys. Rev. E 65, 066614 (2002).

[61] P.G. Kevrekidis and V.V. Konotop, Math. Comput. Simul. 62, 79 (2003).

[62] M. Johansson, S. Aubry, Yu.B. Gaididei, P.L. Christiansen and K.Ø. Rasmussen, Physica D 119, 115 (1998).

[63] B.A. Malomed and P.G. Kevrekidis, Phys. Rev. E 64, 026601 (2001).

[64] D.E. Pelinovsky, P.G. Kevrekidis and D.J. Frantzeskakis, Physica D 212, 20 (2005).

[65] M. Öster and M. Johansson, Phys. Rev. E 73, 066608 (2006).

[66] Yu.S. Kivshar and M. Peyrard, Phys. Rev. A 46, 3198 (1992).

[67] H. Susanto, P.G. Kevrekidis, R. Carretero-González, B.A. Malomed, and D.J. Frantzeskakis, Phys. Rev. Lett. 99, 214103 (2007).

[68] F.Kh. Abdullaev, Yu.V. Bludov, S.V. Dmitriev, P.G. Kevrekidis, and V.V. Konotop, Phys. Rev. E 77, 016604 (2008).

[69] S.V. Dmitriev, P.G. Kevrekidis, N. Yoshikawa, and D.J. Frantzeskakis, J. Phys. A: Math. Theor. 40, 1727 (2007).

[70] A. Khare, S.V. Dmitriev, and A. Saxena, J. Phys. A: Math. Theor. 40, 11301 (2007).

[71] D.E. Pelinovsky, T.R.O. Melvin, and A.R. Champneys, Physica D 236, 22 (2007).

[72] O.F. Oxtoby and I.V. Barashenkov, Phys. Rev. E 76, 036603 (2007).

[73] T.R.O. Melvin, A.R. Champneys, P.G. Kevrekidis, and J. Cuevas, Physica D 237, 551 (2008).

[74] C. Chong, R. Carretero-González, B.A. Malomed, and P.G. Kevrekidis, arXiv:0804.0497v1 [nlin.PS] 3 Apr 2008. 